Andrea Consiglio \& Domenico De Giovanni

Pricing the Option to Surrender in Incomplete Markets

Finance

Research Group 


\title{
Pricing the Option to Surrender in
}

\section{Incomplete Markets}

\author{
Andrea Consiglio * Domenico De Giovanni ${ }^{\dagger \ddagger}$
}

First Draft: August 2007

\author{
Working Paper 07-03 \\ Department of Statistics and Mathematics "Silvio Vianelli" \\ University of Palermo
}

ITALY

\footnotetext{
*Department of Statistics and Mathematics "Silvio Vianelli", University of Palermo, Italy. email: consiglio@unipa.it

${ }^{\dagger}$ Corresponding author. Finance Research Group, Department of Business Studies, Aarhus School of Business, University of Aarhus, Denmark. email: dogi@asb.dk

¥This research was carried out while Domenico De Giovanni was affiliated with University of Palermo.
} 


\title{
Pricing the Option to Surrender in Incomplete Markets
}

\begin{abstract}
New international accounting standards require insurers to reflect the value of embedded options and guarantees in their products. Pricing techniques based on the Black \& Scholes paradigm are often used, however, the hypotheses underneath this model are rarely met.

We propose a framework that encompasses the most known sources of incompleteness. We show that the surrender option, joined with a wide range of claims embedded in insurance contracts, can be priced through our tool, and deliver hedging portfolios to mitigate the risk arising from their positions. We provide extensive empirical analysis to highlight the effect of incompleteness on the fair value of the option.
\end{abstract}

Keywords: Life insurance, Policies with minimum guarantee, Option pricing, Incomplete markets, Surrender options. 


\section{Introduction}

In a recent paper, the International Association of Insurance Supervisors (Solvency and Actuarial Issues Subcommittee, 2006) describes best practices for asset-liability management (ALM) that a well managed insurer would be expected to follow. One of their statements is addressed to embedded options in insurance contracts:

"While the risk of embedded options generally can not be diversified, it is important that insurers manage their assets and liabilities in a way that would mitigate the potential impact. Management might, for example, take actions such as arranging reinsurance, hedging, offsetting with other types of policies, or stopping sales of the product."

The issue of pricing and hedging embedded options in insurance contracts is also ruled in the the International Financial Reporting Standards for insurance contracts (IFRS 4) and Solvency II. The new reporting standard recommends that the costs of options and guarantees embedded in insurance contracts is measured in a market consistent way, and, to achieve market consistency, it is suggested to split the valuation into hedgeable and nonhedgeable components.

If an exposure can be perfectly hedged or replicated on a sufficient liquid and transparent market, the hedge or replicating portfolio provides a directly observable price (marked-to-market). For the non-hedgeable liability component and for the remaining risk on partial hedges, the valuation process would need to rely on methodologies to deliver adequate proxies determined 
on a market consistent basis, i.e. arbitrage-free mark-to-model techniques.

Hence, hedging is the "golden rule" suggested by international regulators, and insurance companies will be judged and ranked according to these aspects.

But, a question arises spontaneously: "Can we always hedge?".

Correctly, regulators subdivide exposures in hedgeable and non-hedgeable components. However, the boundary between hedgeable and non-hedgeable positions is often blurred; moreover, this distinction strongly depends on the model adopted.

For example, insurance guarantees, bonus provisions and other related embedded options can be priced under the Black \& Scholes framework (see Bacinello, 2001; Giraldi et al., 2003; Grosen and Jørgensen, 2000; Hansen and Miltersen, 2002). However, their approach implies that the underlying is a tradable asset, and the option can be replicated by a portfolio of the reference fund and the risk free rate. This is fairly impossible in practice!

This is also unsafe for index-linked policies. For such products, although the underlying can be easily traded using futures or options written on the index, the liabilities generated by the insurance contract are long term ones, and unexpected shortfalls in the asset returns are not envisaged by the brownian motion underneath the Black \& Scholes model. These unpredictable events can generate serious losses. Rolling-the-hedge-forward is not a safe solution, as witnessed by the Metallgesellschaft default (Mello and Parsons, 1995).

To summarize, hedging is suggested as a good practice to mitigate market swings, however, a replicating portfolio is not always available, because of 
either non-tradable assets or too simplistic hypotheses about the market evolution.

In the theory of financial option pricing, such limitations are known as sources of market incompleteness. Recently, scholars have started coping with pricing and hedging options in incomplete markets.

Specifically to the insurance field, Møller (2001) determines risk-minimizing hedging strategies for equity-linked pure endowment contracts, where the source of incompleteness arises from mortality risk.

Moore and Young (2003) employ a utility method to determine the price of endowment contracts linked to risky indexes. In this case too, the source of incompleteness is the mortality risk.

Coleman et al. (2006) deal with the same problem and solve it in a more general setting. In particular, they model the dynamics of the objective price measure by merging the traditional Black \& Scholes price process with the Merton's jump diffusion process. They then hedge the insurance claim using the underlying asset and a set of standard options expiring before the maturity of the claim. The hedging strategy is determined by applying the minimum local hedging risk principle by Föllmer and Schweizer (1989).

Consiglio and De Giovanni (2008) adopt a super-replication model to determine the hedging portfolio of insurance policies whose final liabilities depend on a minimum guarantee option and a bonus distribution scheme. Their model is general enough to deal with any complex final payoff generated by European path-dependent options. They also account for the bankrupt event by considering the liabilities of the company as a risky (defaultable) bond. Regulatory restrictions are modeled by assuming that the solvency of 
the company is monitored at discrete points in time.

The aim of the present paper is to extend to the surrender option the analysis developed in Consiglio and De Giovanni. Our starting point is the approach proposed by Grosen and Jørgensen (1997). They model the surrender option as an American option, since its maturity is unknown at the inception of the contract. We will also investigate the instance where the lapse rate is represented by a stochastic process, functionally dependent on some economic factors. In this case, the surrender option turns to a pathdependent European option, and the incompleteness introduced by the additional risk factor is properly treated through our model.

The paper is organized as follows. In Section 3, we describe the models used to deal with lapses; Section 4 is devoted to the pricing of embedded options under the incomplete market hypothesis. Implementation notes and discussion of the results are reported in Section 6. Section 7 concludes the paper and highlights the major findings.

\section{Preliminaries}

We assume that the security prices are stochastic processes which evolve on a discretized space and time. The market consists of $J$ tradable securities, whose prices are observed on a finite number of decision stages, $t=0,1,2, \ldots, T$

$$
S=\left(S_{t}^{1}, \ldots, S_{t}^{J}\right)_{t=0}^{T}
$$

The price process is modeled on the probability space $(\Omega, \mathcal{F}, P)$, where 
the sample space $\Omega$ is assumed to be finite. Such a formulation allows for a market representation through scenario trees (see Pliska, 1997).

We denote by $\mathcal{N}_{t}$ the set of nodes at stage $t$. Each node $n \in \mathcal{N}_{t}$ corresponds, one-to-one, with an atom of the filtration $\mathcal{F}_{t}$. In the tree, every node $n \in \mathcal{N}_{t}, t=1, \ldots, T$, has a unique ancestor node $a(n) \in \mathcal{N}_{t-1}$, and every node $n \in \mathcal{N}_{t}, t=0, \ldots, T-1$, has a non-empty set of child nodes $\mathcal{C}(n) \subset \mathcal{N}_{t}$. The collection of all the nodes is denoted by $\mathcal{N} \equiv \bigcup_{t=0}^{T} \mathcal{N}_{t}$.

The probabilistic structure implies that the market evolves as a discrete, non-recombinant tree (hence, suitable for incomplete markets). In this context, a European contingent claim (ECC) is a security associated to the stochastic process $S=\left(S_{t}\right)_{t=0}^{T}$, that gives to its owner a stochastic cashflow $F=\left(F_{t}\right)_{t=0}^{T}$. An American contingent claim (ACC) is a security associated to the real-valued stochastic process $E=\left(E_{t}\right)_{t=0}^{T}$ that gives to its owner the right to withdraw $E_{t}$ euros, after which the security expires.

The value of $S_{t}, F_{t}$ and $E_{t}$, in each node $n \in \mathcal{N}_{t}$, is denoted by $\left\{S_{n}\right\}_{n \in \mathcal{N}_{t}}$, where $S_{n}=\left(S_{n}^{1}, \ldots S_{n}^{J}\right) \in \mathbb{R}^{J},\left\{F_{n}\right\}_{n \in \mathcal{N}_{t}}$ and $\left\{E_{n}\right\}_{n \in \mathcal{N}_{t}}$, where $F_{n}, E_{n} \in \mathbb{R}$, respectively.

The definitions of ECC and ACC given above are quite general to include put and call options with barriers, lookback and Asian payoff, futures and any derivatives. Note that such definitions cover claims written on multiple underlyings or non-tradeable instruments.

One of the sources of incompleteness is represented by the presence of more risk factors than available instruments. The main consequence is that not all payoffs are replicable by trading in marketed securities. For example, mortality risk or lapse dynamics can be modeled as stochastic processes, 


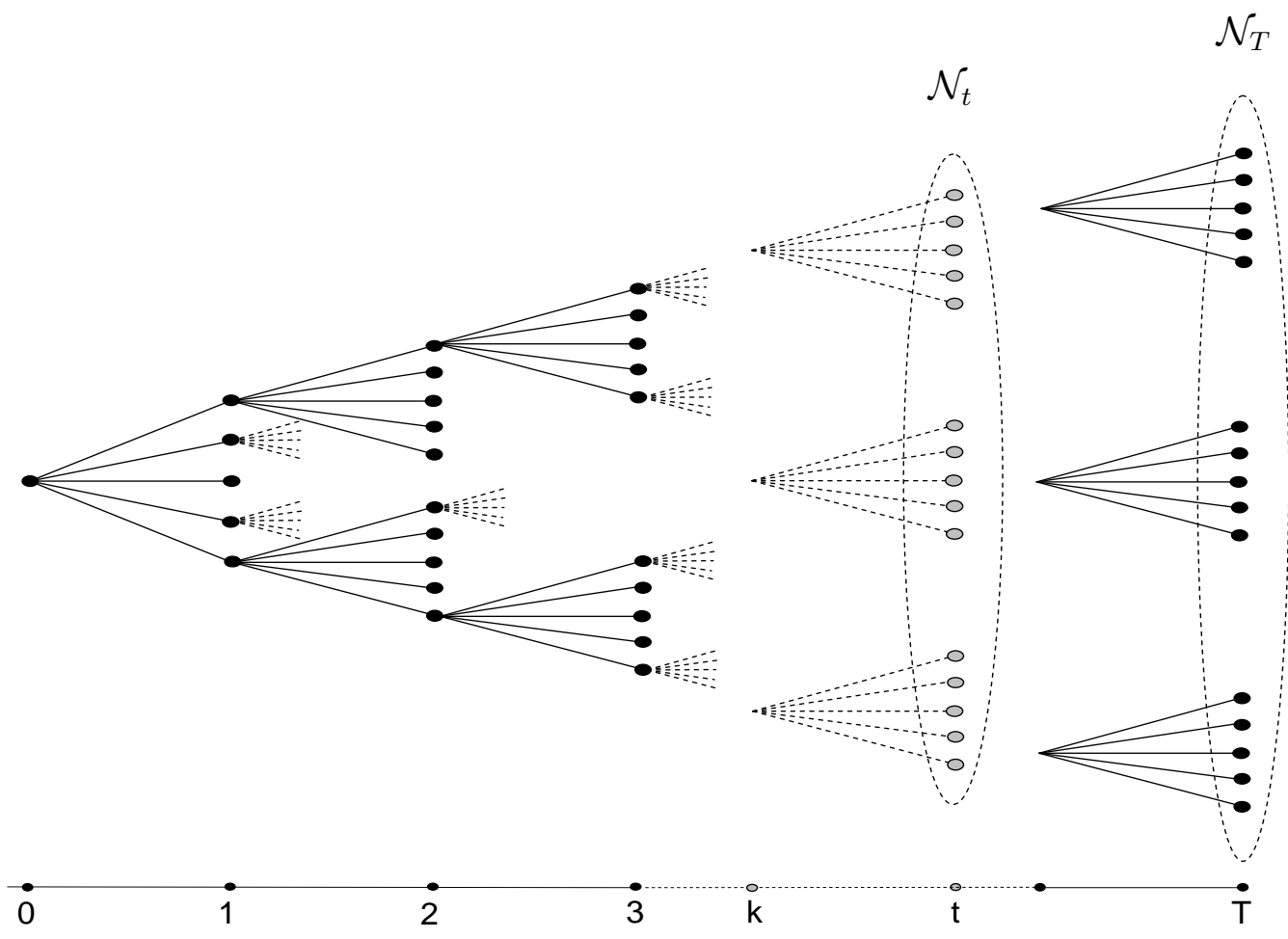

Figure 1: A graphic representation of a non-recombinant tree.

and liabilities which are triggered by such events give rise to contingencies that cannot be perfectly hedged or reached. In practice, the process $S_{t}$ is augmented by $K$ real-valued variables $\xi_{t}=\left(\xi_{t}^{1}, \ldots, \xi_{t}^{K}\right)$ whose path histories match the nodes $n \in \mathcal{N}_{t}$, for each $t=0,1,2, \ldots, T$.

\section{$3 \quad$ Modeling lapses}

The literature on modeling lapses can be subdivided into two strands: one relates the lapse behavior to economic and/or social factors, like, unemployment rate, interest rate, age, education, etc. This approach has been widely used to shape the lapse process for mortgage-backed securities. The other stream describes the lapse process as an internal decision process, where the 
investor rationally exercises his right to surrender the policy.

While the former approach allows for some form of irrationality in lapsing a policy by introducing a minimal lapse rate in each state of the economy, the latter approach identifies a boundary which separates those states of the economy in which it is convenient to sell-back the policy, from those in which it is convenient to keep holding it.

Both approaches have pros and cons. In Albizzati and Geman (1994), the lapse behavior is given by a deterministic (piecewise linear) function of a decision criterion that, in its turn, depends on the stochastic interest rate. In this case, the lapse rate does not introduce a new risk factor, so it can be simply added to the nodes of the tree, $\left\{l_{n}\right\}_{n \in \mathcal{N}_{t}}$, for each $t=$ $1,2, \ldots, T-1$, where $l_{n}=f\left(D_{n}\right)$ and $D_{n}$ depends on the interest rate. The function $f(\cdot)$ is nondecreasing and piecewise linear, and its codomain is $\left[l_{\min }, l_{\max }\right]$. Albizzati and Geman assume that the insurance company has at its disposal a large pool of homogenous policies, and by the principle of pooling-risk, the optimal stopping time transforms into a deterministic aggregate maturity. This implies that the option is an ECC path-dependent option with expected outflows, due to lapses, at fixed dates.

The Albizzati and Geman approach has the main advantage of turning a complex ACC model into a simpler ECC one. However, the lapse mechanism appears to be too simple to be realistic.

A more robust model is described in Kuo et al. (2003). They estimate an error-correction model with two cointegrating vectors of the lapse rate $\left(l_{t}\right)$, interest rate $\left(i_{t}\right)$, and unemployment rate $\left(u_{t}\right)$. By letting $\xi_{t}=\left(\Delta l_{t}, \Delta i_{t}, \Delta u_{t}\right)^{\prime}$, 
and $s_{t}=\left(l_{t}, i_{t}, u_{t}\right)^{\prime}$, they obtain the following,

$$
\xi_{t}=c+A \xi_{t-1}+\alpha \beta s_{t-1}+\varepsilon_{t}
$$

where $c, A, \alpha$, and $\beta$ are parameters to be estimated, together with the variance-covariance matrix of the error, $\Sigma$.

As pointed out in Pennanen (2007), the time series model (2) falls in the general class of the so-called conditional Gaussian processes,

$$
\xi_{t}=\mu\left(\xi_{1}, \ldots, \xi_{t-1}\right)+\sigma\left(\xi_{1}, \ldots, \xi_{t-1}\right) \varepsilon_{t}
$$

In particular, for model (2), we have that

$$
\begin{aligned}
& \mu\left(\xi_{1}, \ldots, \xi_{t-1}\right)=c+A \xi_{t-1}+\alpha \beta s_{t-1}, \quad \text { and } \\
& \sigma\left(\xi_{1}, \ldots, \xi_{t-1}\right)=\Sigma .
\end{aligned}
$$

Once the parameters of the model (2) are estimated, a moment matching method (see Section 5 for details) can be used to build the tree with expected values and variance-covariance matrix given by (4) and (5), respectively.

Using the pooling-of-risk principle, we can thus price the surrender option as an ECC. However, note that, unlike the Albizzati and Geman's model, we introduced here an additional independent risk factor which makes the market incomplete. Also note that the lapse option cannot be hedged as in the Black \& Scholes model, since its underlying - the lapse rate - is a nontradable risk factor.

If the pooling-of-risk principle cannot be invoked, the surrender option 
is a typical ACC. Grosen and Jørgensen (1997) first analyze early exercise options embedded in unit-link insurance contracts, and then extend their contribution to policies with a smoothing surplus distribution mechanism (Grosen and Jørgensen, 2000).

As we will show in Section 4, the framework proposed by Grosen and Jørgensen can be widened to include the hypotheses of market incompleteness discussed in the Introduction.

\section{$4 \quad$ Pricing and hedging in incomplete markets}

Our starting point is the policy described in Grosen and Jørgensen (1997). Our choice is aimed at providing a simple framework to highlight the main findings of our model with respect to the complete market paradigm. We stress here that more realistic bonus schemes and participating formulas can be easily customized (see Consiglio and De Giovanni, 2008, for details).

We assumed that an insurance company issues contracts that promise to pay some benefits, at any time $t \in[0, T]$, contingent to the value of a reference fund $I_{t}$. Note that we do not make any assumptions about the dynamics of $I_{t}$.

We denote by $I_{0}$ the value of the fund at the inception of the contract, and we let $L_{0} \equiv \alpha I_{0}$ be the premium paid by the policyholders to enter the contract; the initial investments by the equityholders are then given by $E_{0} \equiv(1-\alpha) I_{0}$. To be consistent with Grosen and Jørgensen (1997), we set $\alpha=1$, therefore, the initial investment in the reference fund fully comes from the premium paid by the policyholders, $I_{0}=L_{0}$. 
The insurance contract is equipped with a minimum guarantee provision. In particular, at any time period, the policyholder is guaranteed to receive an amount of money, $L_{t}$, obtained by compounding the initial premium, $L_{0}$, at the rate $r_{G}$

$$
L_{t}=L_{0} e^{r_{G} t}
$$

Given an exercise date $t \in[0, T]$, the insurance company is liable for either the interest rate guarantee or the claim of the index market value. The value of the claim is thus given by

$$
\begin{aligned}
C(t) & =\max \left\{L_{t}, I_{t}\right\}= \\
& =\max \left\{L_{0} e^{r_{G} t}-I_{t}, 0\right\}+I_{t} .
\end{aligned}
$$

Relation (7) defines the payoff of a claim on the reference fund, plus an American put option with a strike increasing at the rate $r_{G}$.

The model presupposes that in aggregate the whole class of policyholders exercise the claim and that the option expires. This is a strong assumption, but it could be a realistic approximation in case of high interest rates or unemployment rates. As seen in Section 3, a possible alternative is to introduce an additional risk factor - the lapse process - and price the option as an ECC in the incomplete market framework.

Nothing change from the policyholder's point of view. If the option is exercised, she will receive the greater of either $I_{t}$ or $L_{t}$. From the insurer's point of view, the aggregate liability in the balance sheet is modified to take 
into account the percentage of policyholders who lapsed the contract,

$$
L_{t}=\left(1-l_{t}\right) L_{t-1} e^{r_{G}},
$$

where $l_{t}$ is the lapse rate during the period $t-1$ to $t$. The insurance company will be subjected to an outflow at each time step $t$ given by,

$$
O_{t}=l_{t} L_{t-1} e^{r_{G}}
$$

and the claim will expire at the end of the horizon, $T$.

\subsection{Super-replicating an ECC}

Following King (2002) and Consiglio and De Giovanni (2008), we implement a stochastic programming model to super-replicate the payout process, $\left\{F_{n}\right\}_{n \in \mathcal{N}_{t}}, t=0,1,2, \ldots, T$, generated by an insurance contract where the lapse dynamics is represented by the process $\left\{l_{n}\right\}_{n \in \mathcal{N}_{t}}$, for each $t=$ $1,2, \ldots, T-1$. In particular, we have that

$$
\begin{array}{ll}
F_{n}=\left[L_{n}-I_{n}\right]^{+}+I_{n}, & \text { for all } n \in \mathcal{N}_{T}, \\
F_{n}=l_{n} L_{a(n)} e^{r_{G}}, & \text { for all } n \in \mathcal{N}_{t}, \\
& t=1,2, \ldots, T-1 \\
L_{n}=\left(1-l_{n}\right) L_{a(n)} e^{r_{G}} & \text { for all } n \in \mathcal{N}_{t}, \\
& t=1,2, \ldots, T,
\end{array}
$$

with the initial condition at the root node, $F_{0}=0$ and $L_{0}=I_{0}$. 
In incomplete markets, the price of the option is not unique, and the interval between the buyer's and seller's prices describes the possible range of arbitrage-free evaluations. Since the main aim of this paper is to find an hedging portfolio to mitigate the insurance company risk, we focus our attention on the seller's price.

In an ECC, the seller receives an amount, $V$, and he agrees to pay the buyer the payoff, $F$. The seller's objective is to select a portfolio process of tradable assets that enables her to meet hers obligations without risk (i.e., on all nodes $n \in \mathcal{N})$. The portfolio process must be self-financing, i.e. the amount of assets bought must be offset by the amount of assets sold.

Using the tree-notation, we denote by $Z_{n}^{j}$ the number of shares held at each node $n \in \mathcal{N}$, and for each security $j=1,2, \ldots, J$.

At the root node, the value (price) of the hedging portfolio plus any payout to be covered is the price of the option, that is

$$
\sum_{j=1}^{J} S_{0}^{j} Z_{0}^{j}+F_{0}=V .
$$

At each node $n \in \mathcal{N}_{t}, t=1,2, \ldots, T$, the self-financing constraints are defined as follows

$$
\sum_{j=1}^{J} S_{n}^{j} Z_{n}^{j}+F_{n}=\sum_{j=1}^{J} S_{n}^{j} Z_{a(n)}^{j}
$$

To sum up, the stochastic programming model for the insurance evalua- 
tion problem can be written as follows

$$
\begin{aligned}
& \underset{Z_{n}^{j}}{\operatorname{Minimize}} V \\
& \sum_{j=1}^{J} S_{0}^{j} Z_{0}^{j}+F_{0}=V
\end{aligned}
$$

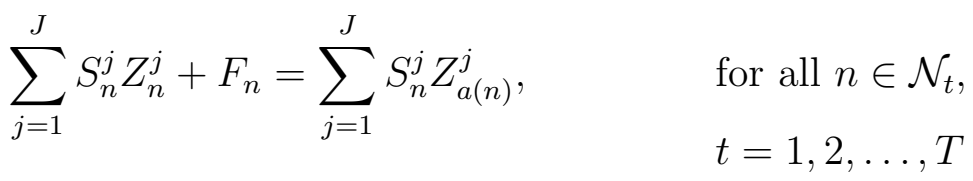

$$
\begin{aligned}
& \sum_{j=1}^{J} S_{n}^{j} Z_{n}^{j} \geq 0, \quad \text { for all } n \in \mathcal{N}_{T}, \\
& Z_{n}^{j} \in \mathbb{R} . \quad \text { for all } n \in \mathcal{N} \text {, } \\
& j=1,2, \ldots, J
\end{aligned}
$$

We highlight here some important points:

1. Problem (15)-(19) is a linear programming model where the objective function minimizes the value of the hedging portfolio, or rather the price of the option.

2. The payout process $\left\{F_{n}\right\}_{n \in \mathcal{N}}$ is a parameter of problem (15)-(19). This implies that any complicated structure for $F_{n}$ does not change the complexity of the model. In our example, the process $\left\{F_{n}\right\}_{n \in \mathcal{N}}$ is defined by relations $(10)-(12)$.

3. Constraints (18) ensure that at each final node the total position of the hedging portfolio is not short. In other words, if short positions are allowed, the portfolio process must end up with enough long positions so that a positive portfolio value is delivered. 


\subsection{Super-replicating an ACC}

Pennanen and King (2005) provide a formal framework to derive the range of prices for an ACC. As for the ECC, we need to distinguish between the seller's and buyer's price. For an ACC, the buyer's price is obtained by hedging against some stopping time. This introduces binary variables to keep track of the past history. However, Pennanen and King show that the buyer's price can be determined as the optimum value of a convex (indeed, a linear program) minimization problem. The seller's price is technically simpler to solve. In fact, the seller needs to hedge against any stopping time. To this purpose, we modified problem (15)-(19) by introducing a set constraints such that at each node the portfolio value reaches at least the payout process $E$.

In the tree-notation, for each $n \in \mathcal{N}_{t}, t=0,2, \ldots, T$, we have that

$$
\sum_{j=1}^{J} S_{n}^{j} Z_{n}^{j} \geq E_{n}
$$

where

$$
\begin{array}{ll}
E_{n}=\left[L_{n}-I_{n}\right]^{+}+I_{n}, & \text { for all } n \in \mathcal{N}_{t}, \\
& t=0,1,2, \ldots, T \\
L_{n}=L_{0} e^{r_{G} t}, & \text { for all } n \in \mathcal{N}_{t}, \\
& t=0,1,2, \ldots, T .
\end{array}
$$

Note that the self-financing constraints are also modified, since the hedging process has to delivery a value at least equal to $E_{n}$. 
In summary, the seller's price is determined by the following linear program

$$
\begin{array}{ll}
\underset{Z_{n}^{j}}{\operatorname{Minimize}} V & \\
\sum_{j=1}^{J} S_{0}^{j} Z_{0}^{j}=V, & \\
\sum_{j=1}^{J} S_{n}^{j} Z_{n}^{j}=\sum_{j=1}^{J} S_{n}^{j} Z_{a(n)}^{j}, & \text { for all } n \in \mathcal{N}_{t}, \\
\sum_{j=1}^{J} S_{n}^{j} Z_{n}^{j} \geq 0, & t=1,2, \ldots T \\
\sum_{j=1}^{J} S_{n}^{j} Z_{n}^{j} \geq E_{n}, & \text { for all } n \in \mathcal{N}_{T}, \\
& \text { for all } n \in \mathcal{N}_{t}, \\
Z_{n}^{j} \in \mathbb{R} . & t=0,1, \ldots T, \\
& \text { for all } n \in \mathcal{N}, \\
& j=1,2, \ldots, J
\end{array}
$$

\section{The tree generation model}

We generate the tree of the underlying price process $S$ by matching the first $M$ moments of its unknown distribution. Our approach is based on the model by Høyland and Wallace (2001), where the user provides a set of moments, $\mathcal{M}$, of the underlying distribution (mean, variance, skewness, covariance, or quantiles), and then, prices and probabilities are jointly determined by solving a nonlinear system of equations, or a nonlinear optimization problem. The method also allows for intertemporal dependencies, such as mean reverting or volatility clumping effect. 
For a review on alternative scenario generation methods, see Dupačová et al. (2000) and references therein.

We cast the tree generation model as a non-convex weighted least squares minimization problem

$$
\begin{aligned}
\underset{S, p}{\operatorname{Minimize}} & \sum_{j=1}^{J}\left\{\sum_{i \in \mathcal{M}} \alpha_{i}\left[\sum_{m \in \mathcal{C}(n)} p_{m}\left(\ln \frac{S_{m}^{j}}{S_{n}^{j}}\right)^{i}-\mu_{i}^{j}\right]^{2}\right\}+ \\
& \sum_{\substack{j \\
h>j}}^{J} \sum_{h}^{J}\left\{\gamma_{j h}\left[\sum_{m \in \mathcal{C}(n)} p_{m}\left(\ln \frac{S_{m}^{j}}{S_{n}^{j}}-\mu_{1}^{j}\right)\left(\ln \frac{S_{m}^{h}}{S_{n}^{h}}-\mu_{1}^{h}\right)-\rho\right]^{2}\right\}
\end{aligned}
$$

$$
\begin{aligned}
\text { s.t. } \quad \sum_{m \in \mathcal{C}(n)} p_{m} & =1 \\
p_{m} & \geq 0 \quad m \in \mathcal{C}(n) .
\end{aligned}
$$

The goal programming model (29)-(31) is much more flexible than solving a system of nonlinear equations matching the moments of the unknown distribution. As underlined in Høyland and Wallace (2001), the solvability of the nonlinear system of equations increases with the number of arcs. However, to be tractable, the set of arcs that springs out from each node has to be of limited size. In fact, the size of the tree grows exponentially with the number of branches springing out from each node. In particular, if $|\mathcal{C}(n)|=\nu$, the total number of nodes is $\sum_{t} \nu^{t}+1$. 
Note, however, that infeasibility could also arise from an inconsistency in the specification of the moments (see Høyland and Wallace, 2001 for a discussion on this topic).

\subsection{Non-arbitrage constraints}

To be consistent with financial asset pricing theory, the tree has to exclude arbitrage opportunities. This is a very important feature, since in the presence of arbitrage scenarios the model (15)-(19) will end up with an unbounded solution.

Arbitrage opportunities can be avoided either by adding non-arbitrage constraints or by increasing the number of arcs. The latter solution is often impracticable due to the exponential growth of the tree size.

Following Klaassen (2002), it is possible to prove that arbitrage opportunities of the first type are prevented if and only if

$$
\begin{aligned}
\pi_{n} S_{n}^{j}-\sum_{m \in \mathcal{C}(n)} \pi_{m} S_{m}^{j} & =\sum_{m \in \mathcal{C}(n)} p_{m} S_{m}^{j} \quad \text { for all } j=1, \ldots J \\
\pi_{m} & \geq 0 \quad m \in \mathcal{C}(n) \\
\pi_{0} & \in \mathbb{R} .
\end{aligned}
$$

Likewise, arbitrage opportunities of the second type are excluded if and 
only if

$$
\begin{gathered}
\sum_{m \in \mathcal{C}(n)} \nu_{m} S_{m}^{j}=S_{n}^{j} \quad \text { for all } j=1, \ldots J \\
\nu_{m} \geq 0 \quad m \in \mathcal{C}(n) .
\end{gathered}
$$

The set of equations (32)-(34) and (35)-(36) can be added as constraints to the goal programming model (29)-(31) to preclude arbitrage opportunities of both types in the tree that is generated. Note that equations (32) and (35) are nonlinear constraints and must be handled with care. In our experiments we added only first type arbitrage constraints, and those were sufficient to guarantee trees without arbitrage opportunities.

\section{$6 \quad$ Implementation notes and results}

We are now ready for the empirical testing of the model. We first assumed a complete market, i.e. a market where the number of instruments available is equal to the sources of risk. We calibrated the tree by using model (29)(31), and restricted the matching problem to constant means, variances and covariances, with kurtosis $\beta_{2}=3$ and skewness $\beta_{1}=0$. We run our experiments assuming a policy horizon of $T=10$ years, and a time interval between two periods set to 1.67 years, for a total of 6 time periods. Without loss of generality, we assumed the initial prices $S_{0}^{j}=100$, for all $j=1,2, \ldots, J$, and the initial liability $L_{0}=100$.

In Table 1 we display the statistical properties for each of the $J$ assets 


\begin{tabular}{lrcrrrrr}
\hline & Mean & St Dev & \multicolumn{5}{c}{ Correlations } \\
\hline & & & Asset-1 & Asset-2 & Asset-3 & Asset-4 & Ref. Fund \\
\hline Asset-1 & 0.04 & 0.13 & 1 & & & & \\
Asset-2 & 0.045 & 0.15 & 0.6 & 1 & & & \\
Asset-3 & 0.054 & 0.2 & 0.45 & 0.24 & 1 & & \\
Asset-4 & 0.08 & 0.25 & 0.1 & 0.08 & 0.1 & 1 & \\
Ref. Fund & 0.03 & 0.1 & 0.33 & 0.21 & 0.12 & 0.04 & 1 \\
\hline
\end{tabular}

Table 1: Statistical properties of the securities used in the experiments. We built trees by matching the means, variances and covariances shown in the table. All the data are on annual basis.

and the reference fund ${ }^{1}$.

We then released the completeness hypotheses and computed the option price and hedging portfolio. In particular, we studied the case of departure from the gaussian distribution, and the case of additional risk factors, like the lapse rate. As a benchmark, we priced the American put option using the model proposed by Grosen and Jørgensen (1997).

In Figure 2, we show the prices of an $\mathrm{ACC}$ computed according to the model (23)-(28) and the corresponding benchmark prices. In complete markets we expect that both models deliver the same price. We observe a slight difference between the two prices (the highest absolute percentage change is less than 10\%). We ascribe such differences to numerical approximations. However, a possible explanation could also be that the market employed is quasi-complete. In particular, we are working with discrete time steps (1.6 years), and we are not using the underlying asset to hedge the option. The latter choice is motivated by the issue of hedging with non-tradable under-

\footnotetext{
${ }^{1}$ To be consistent with Grosen and Jørgensen, we assumed the risk free rate is constant at the level of $2.5 \%$ per year. Stochastic interest rates can be easily modeled, see Consiglio and De Giovanni (2008).
} 


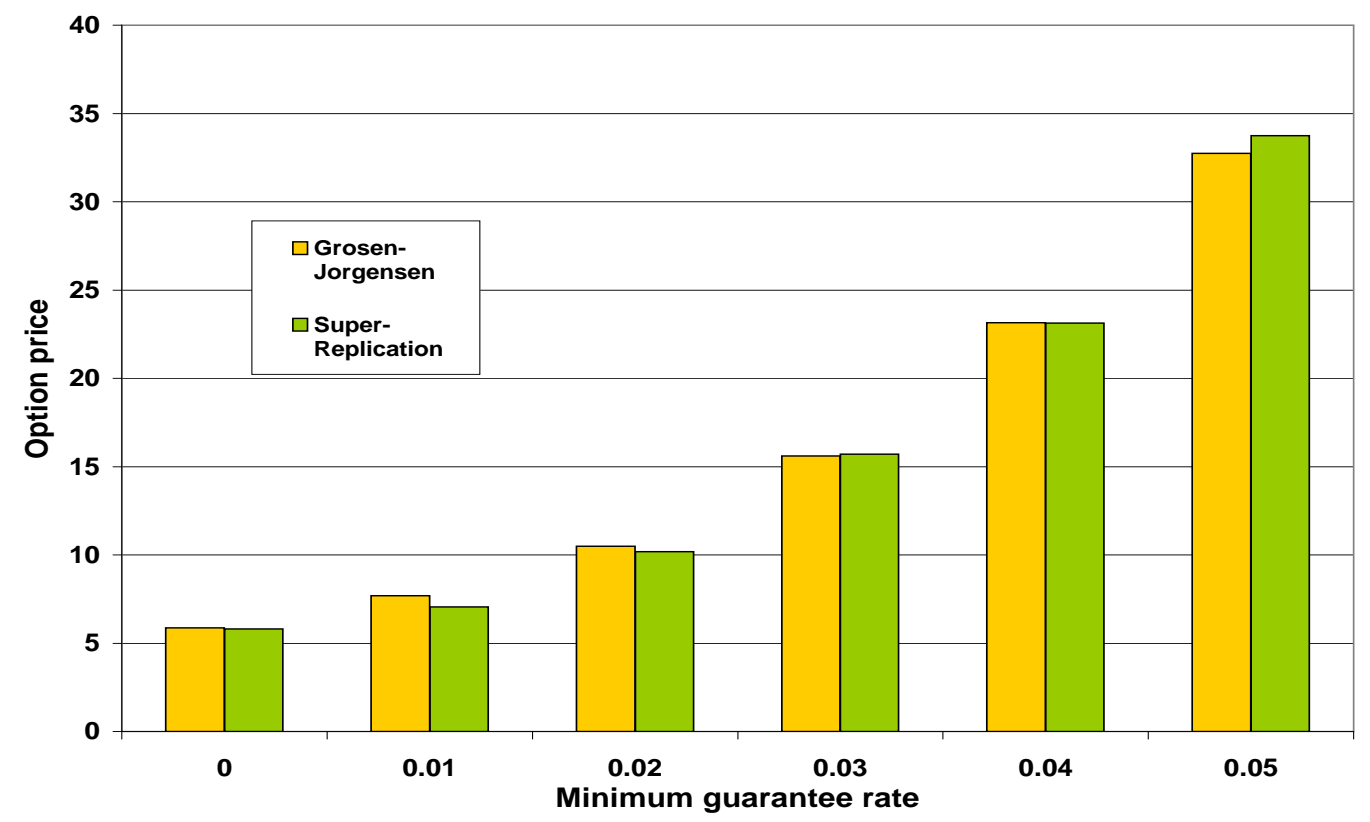

Figure 2: The prices of the ACC insurance contract are determined, respectively, by the super-replication model and the Grosen and Jørgensen's model. The prices are shown for different levels of the minimum guarantee rates. The market is assumed to be complete. 
lyings. The results exhibited show that liabilities triggered by non-tradable assets (in this case, the non-tradable is the reference asset purposely not included among the available securities) can be hedged by a portfolio of liquid assets. We believe this is an advantage upon using proxies to offset nonhedgeable positions.

Our methodology also allows for an investigation of how the option price changes for different hypotheses about the distribution of the underlying assets. As known, the Black \& Scholes model assumes that the asset returns are normal distributed.

We built trees with $\beta_{2}=\{3,4,5,6\}$ and $\beta_{1}=0$. We then priced the ACC originated by the insurance contract given above. We observe that the price of the option with $r_{G}=1 \%$ almost double when moving from $\beta_{2}=3$ gaussian distribution - to $\beta_{2}=6$-fat-tailed distribution (see Figure 3, top). The price interval also depends on the level of the minimum guarantee. In particular, for $r_{G}=4 \%$ (see Figure 3 , bottom), although the magnitude of the option price is higher with respect to $r_{G}=1 \%$, the price interval does not vary across the kurtosis. The reason is that an insurance policy with a $4 \%$ minimum guarantee is deep in-the-money (the risk free rate is $2.5 \%$ ), and therefore the distribution of the assets does not play any role in determining the prices.

In Table 2 we display hedging portfolios and option prices for the given range of minimum guarantee rates and $\beta_{2}=3$. A negative value denotes a short sale. As expected, the shorted option (we are sellers) is hedged by a short portfolio. The proceeds is then invested in the risk free rate (long position). 

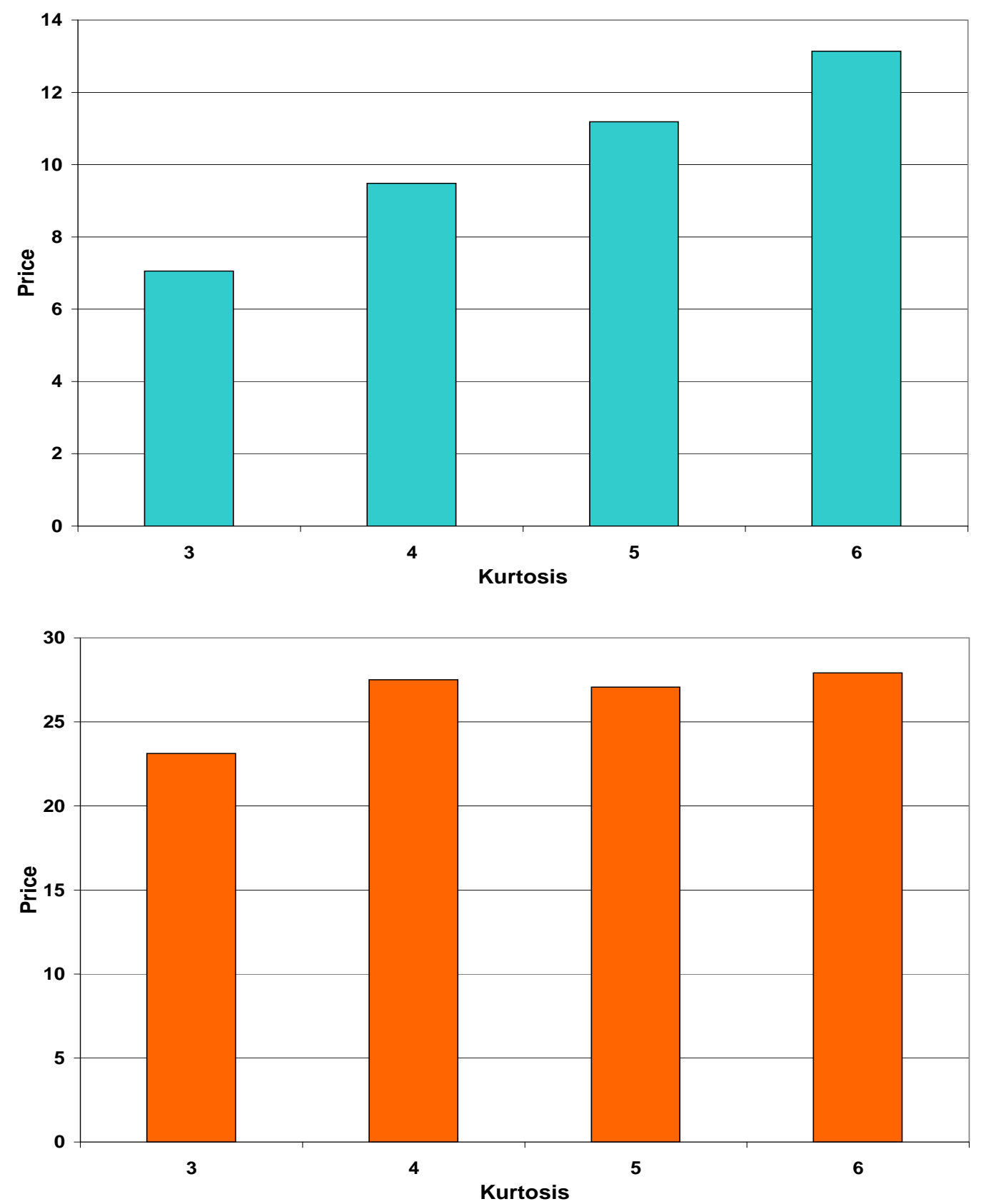

Figure 3: The prices of the ACC insurance contract against asset returns with different kurtosis. The kurtosis has an effect on the magnitude of the option prices. Such an effect also depends on the level of the minimum guarantee. For $r_{G}=1 \%$, lower than the risk free rate (top panel), the kurtosis strongly affects the price of the option. If the option is deeply in-the-money, $r_{G}=4 \%$ (bottom panel), the kurtosis of the asset returns is irrelevant. 
However, as the minimum guarantee increases, and the option becomes in-the-money, the weight of Asset-4 turns positive. Long positions are more evident when the market deviates from completeness. For $\left(\beta_{2}=6\right)$, we observe that even when $r_{G}=0 \%$ long positions for Asset-3 and Asset-4 are held in the portfolio. Our clue is that due to the higher kurtosis, extreme events are more likely in the tree, and to super-replicate the option, a long position in assets with higher expected returns and volatility is less costly than a long position in the risk free only. For in-the-money options, high positive payoffs can be reached more easily with moderate long positions in assets with higher expected returns and volatilities.

As seen in Section 3, the surrender option can be introduced by modeling the lapse dynamics to take into account of the relationship with economic variables. Using such an approach, the claim embedded in the insurance contract is an ECC, and given the additional risk factor, the pricing has to be carried out in the incomplete market framework. From our point of view, this simply implies a modification of the linear programming model described in Section 4.

Since the fitting of an error-correction model is outside the scope of this paper, we assumed that $\mu\left(\xi_{1}, \ldots, \xi_{t-1}\right)=\mu=8 \%$ and $\sigma\left(\xi_{1}, \ldots, \xi_{t-1}\right)=\sigma=$ $1 \%$ per annum. We calibrated a tree by also assuming that the lapse rate is bounded above and below $l_{\min }=5 \%$ and $l_{\max }=10 \%^{2}$ respectively, and set the correlation with the short term interest rate to 0.6.

In Figure 4, we show the prices obtained by modeling the option either as

\footnotetext{
${ }^{2}$ These data are taken from the Life Insurance Fact Book 2006 of the American Council of Life Insurance.
} 


\begin{tabular}{lrrrrrr}
\hline & \multicolumn{7}{c}{$\beta_{2}=3$} \\
\hline Risk free & $0 \%$ & $1 \%$ & $2 \%$ & $3 \%$ & $4 \%$ & $5 \%$ \\
Asset-1 & -3.78 & -6.83 & -9.97 & -14.95 & -18.43 & -22.14 \\
Asset-2 & -5.33 & -7.80 & -10.95 & -15.52 & -21.49 & -25.09 \\
Asset-3 & -2.32 & -1.05 & -1.37 & -1.42 & -0.93 & -1.94 \\
Asset-4 & -0.02 & -0.02 & 0.86 & 1.73 & 2.92 & 2.832 \\
Option Price & -5.55 & -7.29 & -10.59 & -15.84 & -23.07 & -33.66 \\
\hline & \multicolumn{7}{c}{$\beta_{2}=6$} & & \\
\hline & $0 \%$ & $1 \%$ & $2 \%$ & $3 \%$ & $4 \%$ & $5 \%$ \\
\hline Risk free & 21 & 26 & 31 & 40 & 51 & 65 \\
Asset-1 & -9.93 & -12.33 & -14.92 & -18.68 & -24.10 & -29.89 \\
Asset-2 & -8.57 & -9.89 & -11.12 & -13.68 & -17.27 & -21.33 \\
Asset-3 & 4.90 & 6.05 & 7.34 & 9.24 & 12.02 & 14.87 \\
Asset-4 & 2.74 & 3.32 & 3.83 & 4.82 & 6.70 & 8.94 \\
Option Price & -10.62 & -13.24 & -16.55 & -21.68 & -28.79 & -37.69 \\
\hline
\end{tabular}

Table 2: Hedging portfolios and option prices for various levels of the minimum guarantee and kurtosis. Values displayed are obtained by assuming $\beta_{2}=\{3,6\}$. Negative values denote short sales. The proceeds of the short sales is invested in the risk free rate to hedge the option. As the guarantee increases, and the option becomes in-the-money, the weight of Asset-4 turns positive. This occurrence is more evident for fat-tailed returns where high positive payoffs can be reached more easily by long positions in assets with higher expected returns and volatilities. 


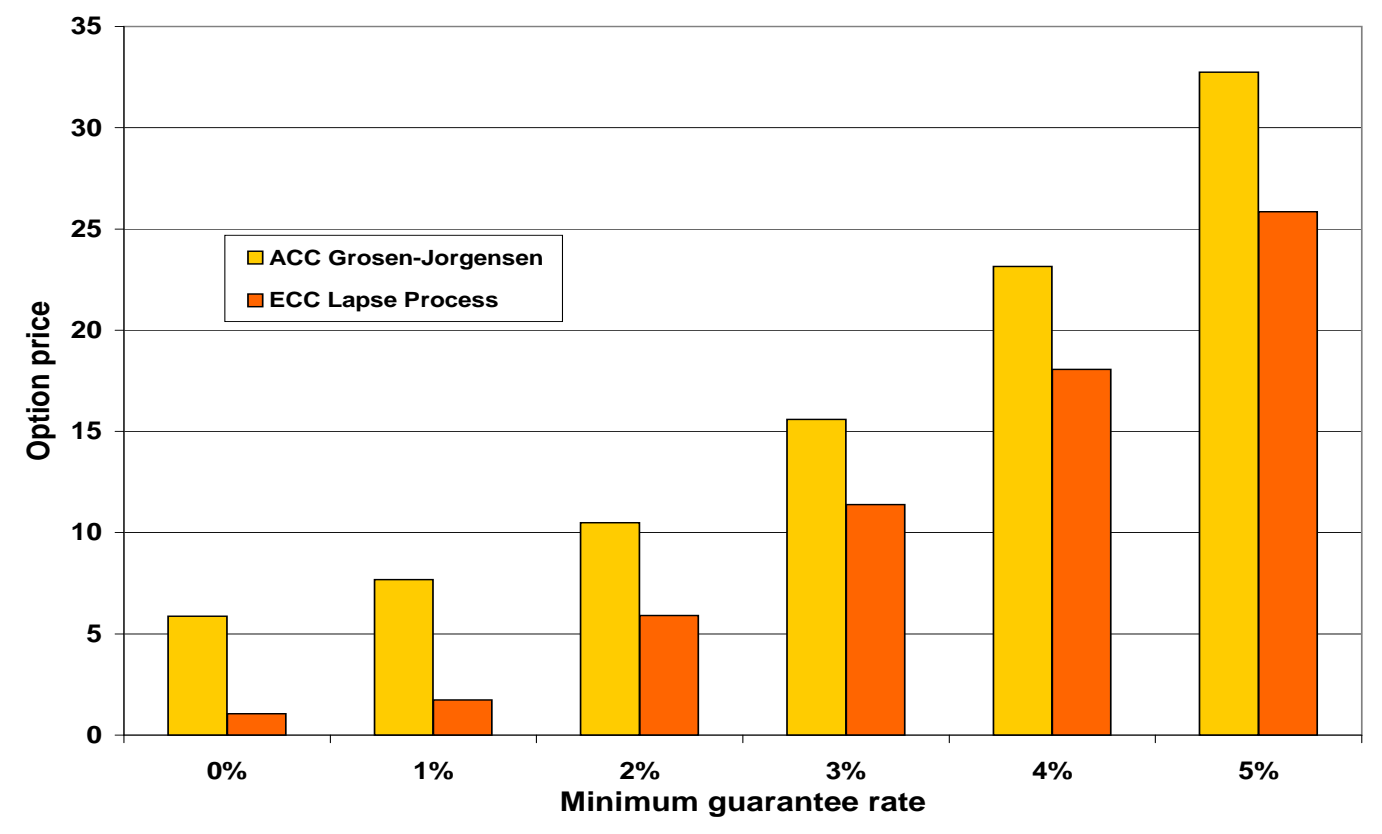

Figure 4: Prices of the surrender option in the complete and incomplete market framework. In the complete case, the surrender option is modeled as an ACC; in the incomplete case, as an ECC. The ECC prices are noticeably lower than the ACC ones, and this is due to a finer representation of the policyholder's lapse behavior.

an ACC using the Grosen and Jørgensen approach, or as an ECC using the incomplete market approach described above. The ECC prices are noticeably lower than the ACC ones. We ascribe such a result to a finer representation of the policyholder's lapse behavior. In particular, the presence of a minimum amount of lapse in each node abates the final level of the option payoff, and consequently, the final liability of the insurance company.

\section{Conclusions}

We have developed a super-replication model to price options embedded in insurance contracts. Several interesting conclusions can be drawn from the 
use of the model. First, new regulations press in the direction of mitigating risk expositions. Our model is able to deliver hedging portfolios even in the worst case situation where the underlying asset is not tradable. We do not make any assumption about the dynamics of the underlying assets. Therefore, any distributional hypothesis can be used to calibrate the stochastic processes. We have also showed that even ACC can be priced using our methodology, thus enlarging the spectrum of possible insurance contracts. We have tested the model using the most classic example of ACC in insurance contracts: the surrender option. The same framework can be adopted either by considering the surrender option as a pure ACC or transforming it in an ECC. We showed that our model improves upon both approaches. In particular, it improves upon the Albizzati and Geman's model, since it allows for richer and more robust modeling of the lapse rate dynamics. It improves the Grosen and Jørgensen's model by providing lower option prices due to a better characterization of the lapse process.

\section{References}

M. O. Albizzati and H. Geman. Interest rate risk management and valuation of surrender option in life insurance policies. Journal of Risk and Insurance, 61(4):616-637, 1994

A.R. Bacinello. Fair pricing of life insurance participating policies with a minimum guarantee. Astin Bulletin, 31(2):275-297, 2001.

T.F. Coleman, Y. Li, and M. Patron. Hedging guarantees in variable annu- 
ities under both equity and interest rate risks. Insurance: Mathematics $\mathcal{E}$ Economics, 38:215-228, 2006.

A. Consiglio and D. De Giovanni. Evaluation of insurance products with guarantee in incomplete markets. Insurance: Mathematics $\& 6$ Economics, 2008. DOI: 10.1016/j.insmatheco.2007.04.005.

J. Dupačová, G. Consigli, and S.W. Wallace. Scenarios for multistage stochastic programs. Annals of Operations Research, 100:25-53, 2000.

H. Föllmer and M. Schweizer. Hedging by sequential regression: An introduction to the mathematics of option trading. Astin Bulletin, 1:147-160, 1989.

C. Giraldi, G. Susinno, G. Berti, J. Brunello, S. Buttarazzi, G. Cenciarelli, C. Daroda, and G. Stamegna. Insurance optional. In A. Lipton, editor, Exotic options: The cutting-edge collection technical papers published in Risk 1999-2003, chapter 35. Risk Books, 2003.

A. Grosen and P.L. Jørgensen. Fair valuation of life insurance liabilities: The impact of interest rate guarantees, surrender options, and bonus policies. Insurance: Mathematics \& Economics, 26:37-57, 2000.

A. Grosen and P.L. Jørgensen. Valuation of early exercisable interest rate guarantees. Journal of Risk and Insurance, 64(3):481-503, 1997.

M. Hansen and K.R. Miltersen. Minimum rate of return guarantees: The danish case. Scandinavian Actuarial Journal, 2002(4):230-318, 2002. 
K. Høyland and S.W. Wallace. Generation scenario trees for multistage decision problems. Management Science, 47(2):295-307, 2001.

A.J King. Duality and martingales: A stochastic programming perspective on contingent claims. Mathematical Programming, Series B, 91:543-562, 2002.

P. Klaassen. Comment on "Generating scenario trees for multistage decision problems". Management Science, 48(11):1512-1516, 2002.

W Kuo, C. Tsai, and C. Chen. An empirical study on the lapse rate: the cointegration approach. Journal of Risk and Insurance, 70(3):489-508, 2003.

S. Mello and E. Parsons. Maturity structure of a hedge matters: lessons from the Metallgesellschaft debacle. Journal of Applied Corporate Finance, 8 (1):106-121, 1995 .

T. Møller. Hedging equity-linked life insurance contracts. North American Actuarial Journal, 5(2):79-95, 2001.

K.S. Moore and V.R. Young. Pricing equity-linked pure endowments via the principle of equivalent utility. Insurance: Mathematics \& Economics, 33: 497-516, 2003.

T. Pennanen. Epi-convergent discretizations of multistage stochastic programs via integration quadratures. Mathematical Programming, 2007. Forthcoming. 
T. Pennanen and A. J. King. Arbitrage pricing of american contingent claims in incomplete markets - a convex optimization approach. Working paper, Helsinki School of Economics, 2005.

S.R. Pliska. Introduction to mathematical finance: discrete time models. Blackwell, Malden,MA, 1997.

Solvency and Actuarial Issues Subcommittee. Standard on asset-liability management. Standard 13, International Association of Insurance Supervisors, October 2006. Available at IAIS website: http://www.iaisweb.org. 


\section{Working Papers from Finance Research Group}

F-2007-02 Andrea Consiglio \& Domenico De Giovanni: Pricing the Option to Surrender in Incomplete Markets.

F-2006-09 Peter Løchte Jørgensen: Lognormal Approximation of Complex Pathdependent Pension Scheme Payoffs.

F-2006-08 Peter Løchte Jørgensen: Traffic Light Options.

F-2006-07 David C. Porter, Carsten Tanggaard, Daniel G. Weaver \& Wei Yu: Dispersed Trading and the Prevention of Market Failure: The Case of the Copenhagen Stock Exhange.

F-2006-06 Amber Anand, Carsten Tanggaard \& Daniel G. Weaver: Paying for Market Quality.

F-2006-05 Anne-Sofie Reng Rasmussen: How well do financial and macroeconomic variables predict stock returns: Time-series and cross-sectional evidence.

F-2006-04 Anne-Sofie Reng Rasmussen: Improving the asset pricing ability of the Consumption-Capital Asset Pricing Model.

F-2006-03 Jan Bartholdy, Dennis Olson \& Paula Peare: Conducting event studies on a small stock exchange.

F-2006-02 Jan Bartholdy \& Cesário Mateus: Debt and Taxes: Evidence from bankfinanced unlisted firms.

F-2006-01 Esben P. Høg \& Per H. Frederiksen: The Fractional Ornstein-Uhlenbeck Process: Term Structure Theory and Application.

F-2005-05 Charlotte Christiansen \& Angelo Ranaldo: Realized bond-stock correlation: macroeconomic announcement effects.

F-2005-04 Søren Willemann: GSE funding advantages and mortgagor benefits: Answers from asset pricing.

F-2005-03 Charlotte Christiansen: Level-ARCH short rate models with regime switching: Bivariate modeling of US and European short rates.

F-2005-02 Charlotte Christiansen, Juanna Schröter Joensen and Jesper Rangvid: Do more economists hold stocks?

F-2005-01 Michael Christensen: Danish mutual fund performance - selectivity, market timing and persistence.

F-2004-01 Charlotte Christiansen: Decomposing European bond and equity volatility. 
ISBN 978-87-788-2233-8

Department of Business Studies

Aarhus School of Business

University of Aarhus

Fuglesangs Allé 4

DK-8210 Aarhus V - Denmark

Tel. +4589486688

Fax +4586150188

www.asb.dk 